\title{
Precision measurement of the muonium hyperfine interaction in mesoporous silica and aerogel
}

\author{
M. H. Dehn $\odot,{ }^{1,2,3,{ }^{*}}$ R. Scheuermann, ${ }^{4}$ P.-X. Wang $\odot,{ }^{5}$ Y. Cao, ${ }^{5}$ M. J. MacLachlan $\odot,{ }^{2,5}$ V. M. Zamarion, ${ }^{6}$ \\ D. G. Fleming, ${ }^{3,5}$ and R. F. Kiefl ${ }^{1,2,3, \dagger}$ \\ ${ }^{1}$ Department of Physics and Astronomy, University of British Columbia, Vancouver, British Columbia, Canada V6T 1Z1 \\ ${ }^{2}$ Stewart Blusson Quantum Matter Institute, University of British Columbia, Vancouver, British Columbia, Canada V6T $1 Z 4$ \\ ${ }^{3}$ TRIUMF, Vancouver, British Columbia, Canada V6T $2 A 3$ \\ ${ }^{4}$ Laboratory for Muon Spectroscopy, Paul Scherrer Institut, CH-5232 Villigen PSI, Switzerland \\ ${ }^{5}$ Department of Chemistry, University of British Columbia, Vancouver, British Columbia, Canada V6T 1Z1 \\ ${ }^{6}$ Institute of Chemistry, University of Sao Paulo, Sao Paulo, CEP 05508-000, Brazil
}

(Received 15 September 2019; accepted 22 December 2020; published 12 January 2021)

\begin{abstract}
Precise measurements of the muonium $(\mathrm{Mu})$ hyperfine interaction versus temperature are reported in a silica aerogel and mesoporous silica SBA-15 using a fast-timing spectrometer to detect the precession frequencies of $\mathrm{Mu}$ in a magnetic field of $1.14 \mathrm{~T}$. The observed signals are a sensitive monitor of dynamics associated with the binding and unbinding of $\mathrm{Mu}$ from the silica surface. Above $100 \mathrm{~K}$ the $\mathrm{Mu}$ is effectively off the surface. Significant differences are observed in the way the lines show motional narrowing, and are attributed to differences in sample morphology. At room temperature the effective mean free path appears longer in SBA-15, suggesting it may offer advantages over aerogel as a source of Mu in vacuum.
\end{abstract}

DOI: 10.1103/PhysRevResearch.3.013029

Muonium ( $\left.\mathrm{Mu}=\left[\mu^{+} e^{-}\right]\right)$may be considered the simplest atom since it is the bound state of an electron and a positive muon, both of which are structureless fundamental particles. As such, its properties provide stringent tests to our understanding of quantum electrodynamics [1-3]. In addition, $\mathrm{Mu}$ may be used to create a beam of low-energy muons by ionization of $\mathrm{Mu}$ in vacuum via two-photon absorption $[4,5]$ and subsequent acceleration of the resulting $\mu^{+}$. Such a beam would enable many experiments spanning the fields of fundamental particle experiments (e.g., muon g-2 [6]) and condensed matter physics, where spin-polarized $\mu^{+}$are used as a sensitive probe of local magnetic properties [7]. Those applications, however, require intense slow muon beams and thus an efficient source for $\mathrm{Mu}$ in vacuum.

It is well known that positive muons injected into silica powder form $\mathrm{Mu}$ which escapes into the intergranular spaces with a high probability [8]. Surprisingly, that happens even at low temperatures if the powder grains are small enough, implying the escape occurs while the $\mathrm{Mu}$ is epithermal [9]. Very similar behavior was found for mesoporous silica samples [10-12]. In recent years, silica aerogels have emerged as the moderator of choice for producing $\mathrm{Mu}$ in vacuum at room temperature since they are self-supporting and can be drilled with micron-sized holes, which improve the yield of $\mathrm{Mu}$ in

\footnotetext{
*mdehn@triumf.ca

${ }^{\dagger}$ kiefl@ triumf.ca
}

Published by the American Physical Society under the terms of the Creative Commons Attribution 4.0 International license. Further distribution of this work must maintain attribution to the author(s) and the published article's title, journal citation, and DOI. vacuum $[6,13]$. However, there is little information about the temperature dependence of $\mathrm{Mu}$ production in aerogels and the role of surface interactions, which control $\mathrm{Mu}$ diffusion in the intergranular space and ultimately the production rate of $\mathrm{Mu}$ in vacuum. Additionally, the transport properties of $\mathrm{Mu}$, a well-established light hydrogen analog [14], in highly porous materials with several distinct length scales is of interest since conventional diffusion equations generally do not apply [15-17].

Here, we report a muon spin rotation $(\mu \mathrm{SR})$ study of a silica aerogel and mesoporous silica (SBA-15) using a fast-timing spectrometer capable of resolving high-frequency $\mathrm{Mu}$ precession signals in a $1.14 \mathrm{~T}$ magnetic field. Precision measurements of the temperature dependence of the isotropic hyperfine coupling $v_{0}$ are interpreted using a simple statistical mechanics model. The SBA-15 exhibits a very different low-temperature behavior compared to the aerogel, which is attributed to Mu confinement inside the well-defined mesopores and in the micropores within the channel walls [18]. At $300 \mathrm{~K}$, the measured isotropic hyperfine interaction in both samples is smaller than the vacuum value $v_{0}^{\mathrm{vac}}=$ 4463.30 MHz [19]. This suppression is lower in SBA-15, suggesting this sample has a longer effective mean free path and that mesoporous materials may have some advantage as a source for $\mathrm{Mu}$ in vacuum.

Mesoporous silica (SBA-15) with a density of $\sim 280 \mathrm{mg} / \mathrm{cm}^{3}$ was synthesized using a hydrothermal method [20]. The Brunauer-Emmett-Teller method [21] gave a specific surface area of $595 \mathrm{~m}^{2} / \mathrm{g}$ and a $7.5 \mathrm{~nm}$ average pore size. The aerogel sample was synthesized following Ref. [22] and had a density of $\sim 180 \mathrm{mg} / \mathrm{cm}^{3}$ and a specific surface area of $896 \mathrm{~m}^{2} / \mathrm{g}$. Prior to the experiment each of the samples was heated for $\sim 48 \mathrm{~h}$ at $\sim 150{ }^{\circ} \mathrm{C}$ in a vacuum better than 
$10^{-3}$ mbar to remove residual moisture from the surfaces. The samples were mounted in Ti cells in a cold finger cryostat, and briefly exposed ( $<15 \mathrm{~min}$ ) to ambient conditions between bake-out and mounting. The $\mu$ SR measurements were carried out in the fast-timing high-field spectrometer HAL-9500 at the Paul-Scherrer-Institute (Villigen, Switzerland). Spin-polarized, short-lived $(\tau=2.2 \mu \mathrm{s})$ muons were implanted into the sample with the initial spin polarization perpendicular to the external field $\mathbf{B}$ (transverse field geometry). The evolution of the muon spin polarization was observed via the time-resolved detection of muon decay positrons, which are emitted preferentially along the muon spin direction at the time of decay (see Ref. [7]). In insulating materials, the muon may capture an electron to form $\mathrm{Mu}$, which gives rise to a distinct set of precession frequencies described by the characteristic spin Hamiltonian

$$
\mathcal{H} / h=\gamma_{e} / 2 \pi \mathbf{S}_{\mathbf{e}} \cdot \mathbf{B}-\gamma_{\mu} / 2 \pi \mathbf{S}_{\mu} \cdot \mathbf{B}+v_{0} \mathbf{S}_{e} \cdot \mathbf{S}_{\mu}
$$

where $\mathbf{S}_{\mu}\left(\mathbf{S}_{e}\right)$ is the muon (electron) spin and $\gamma_{\mu} / 2 \pi=$ $135.5 \mathrm{MHz} / \mathrm{T}\left(\gamma_{e} / 2 \pi=28.02 \mathrm{GHz} / \mathrm{T}\right)$ the muon (electron) gyromagnetic ratio. The energies for the four hyperfine levels are shown in the inset in Fig. 1. For $\mathbf{B}$ larger than $B_{0}=$ $v_{0} / 2 \Gamma_{+}$, the hyperfine field of the muon acting on the electron $\left(B_{0}=0.1585 \mathrm{~T}\right.$ in vacuum), one expects two dominant precession signals, $v_{12}$ and $v_{34}$, corresponding to muon spin-flip transitions for $S_{e}^{z}= \pm \frac{1}{2}$,

$$
\begin{aligned}
& v_{12}=\frac{1}{2} v_{0}+\Gamma_{-} B-\frac{1}{2} v_{0}\left[1+\left(B / B_{0}\right)^{2}\right]^{1 / 2}, \\
& v_{34}=\frac{1}{2} v_{0}-\Gamma_{-} B+\frac{1}{2} v_{0}\left[1+\left(B / B_{0}\right)^{2}\right]^{1 / 2},
\end{aligned}
$$

where $\Gamma_{ \pm}=\left(\left|\gamma_{e}\right| \pm\left|\gamma_{\mu}\right|\right) / 4 \pi$. Crucially, the relation

$$
v_{0}=v_{12}+v_{34}
$$

holds at all fields [23], allowing for a precise and direct measurement of the isotropic hyperfine (Fermi contact) interaction $v_{0}$.

An example frequency spectrum for SBA-15 in $1.14 \mathrm{~T}$ $\left(=7.2 B_{0}\right)$ at $300 \mathrm{~K}$ is shown in Fig. 1(a). Both $\mathrm{Mu}$ frequencies $v_{12}$ and $v_{34}$ are well resolved. The frequency labeled $v_{L}$ is attributed to muons that do not form $\mathrm{Mu}$ and therefore precess at the Larmor frequency of the applied field. In both samples, sharp narrow lines are evident at $300 \mathrm{~K}$ [Figs. 1(b) and 1(c), black]. As the temperature is lowered, the Mu lines broaden and shift to lower frequencies by up to $\sim 12 \mathrm{MHz}$ at $2 \mathrm{~K}$ (red). The low-temperature $\mathrm{Mu}$ lines are considerably broader in SBA-15 than in the aerogel, and show indications of a more complicated line shape. All spectra were fit in the time domain to a sum of three exponentially damped cosines using the analysis program MUSRFIT [24-27]. Using Eq. (3), $v_{0}(T)$ is determined for both samples (Fig. 2).

The temperature dependence observed in the aerogel allows for a simple interpretation [28]. Below $10 \mathrm{~K}$, the $\mathrm{Mu}$ is weakly bound to the silica surfaces, which perturbs the Mu $1 s$ orbital, reducing the isotropic part of the hyperfine interaction. With increasing temperature, the $\mathrm{Mu}$ desorbs and undergoes cycling on and off the surface, leading to a weighted time-average of hyperfine frequencies on $\left(v_{s}\right)$ and off $\left(v_{f}\right)$ the surface. At $300 \mathrm{~K}$, the measured hyperfine interaction approaches a constant value $v_{f}$ which is slightly below that of
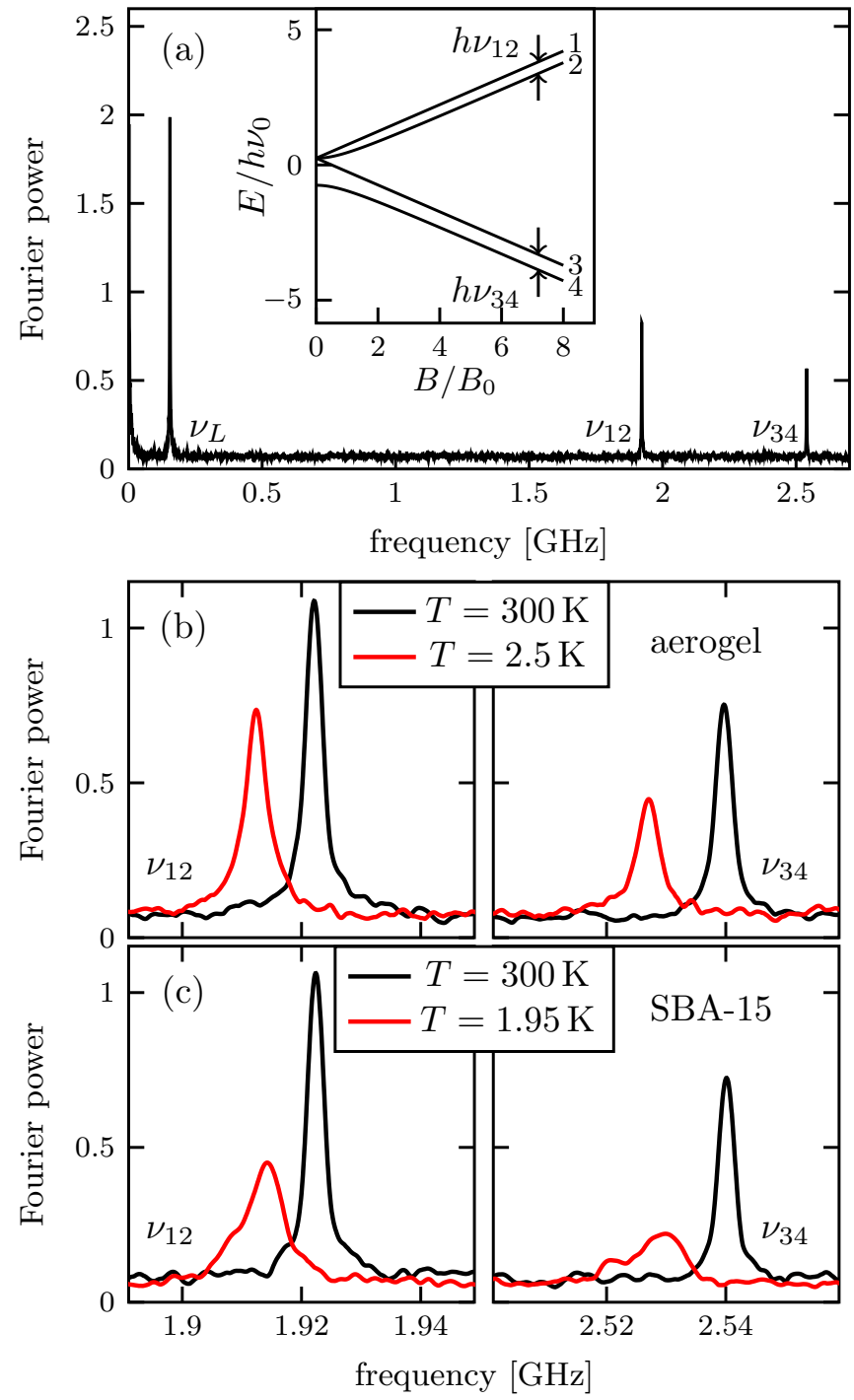

FIG. 1. (a) Fourier transform of the precession signals in SBA-15 near $300 \mathrm{~K}$. The two higher frequencies $\left(v_{12}\right.$ and $\left.v_{34}\right)$ originate from $\mathrm{Mu}$ in the mesopores and intergranular regions. The difference in amplitudes is due to the finite time resolution of the detector system (80 ps). (b) and (c) show expanded regions of the frequency spectrum in the vicinity of $v_{12}$ and $v_{34}$ for both samples. Inset in (a): BreitRabi energy diagram showing energies of the four hyperfine levels vs magnetic field.

$\mathrm{Mu}$ in vacuum. This negative shift is attributed to Mu colliding freely with the silica surfaces, which perturbs the electronic wave function of $\mathrm{Mu}$ during the collision [29,30].

The solid curves in Fig. 2 are a fit to a statistical mechanics model for $\mathrm{Mu}$ confined to a box of volume $V$ with surface area $A, S_{0}$ binding sites per unit area, and a total of $N_{s}=S_{0} A$ surfaces states, each with a binding energy $E_{B}$ [28,31]. The single-particle partition function for such a system is

$$
Z=N_{s} \exp \left(E_{B} / k_{B} T\right)+V\left(m k_{B} T / 2 \pi \hbar^{2}\right)^{3 / 2},
$$

where the first term accounts for the surface bound states and the second for unbound states of $\mathrm{Mu}$ with mass $m$ moving freely. At high enough temperatures where the system cycles 


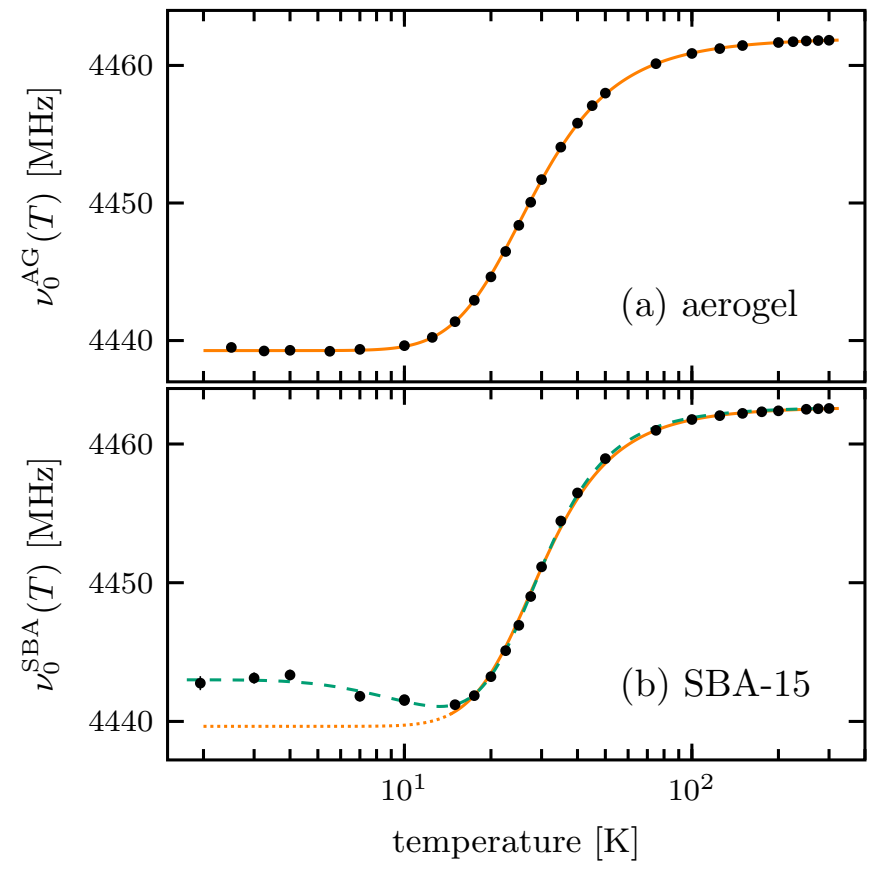

FIG. 2. Hyperfine frequencies as a function of temperature for (a) the aerogel and (b) SBA-15. Orange curves are fits to a two-state model [Eq. (5)] (see Table I). The SBA-15 data was fit between 15 and $300 \mathrm{~K}$ (the dotted line extrapolates outside the fit region). The dashed green line represents a three-state model.

fast through all microstates we expect to observe a mean hyperfine frequency [28],

$$
\begin{aligned}
v_{0}(T) & =\left[v_{s} N_{s} \exp \left(E_{B} / k_{B} T\right)+v_{f} V\left(m k_{B} T / 2 \pi \hbar^{2}\right)^{3 / 2}\right] / Z \\
& =[1-\alpha(T)] v_{f}+\alpha(T) v_{s},
\end{aligned}
$$

where $\alpha(T)=1 /\left[1+\zeta T^{3 / 2} \exp \left(-E_{B} / k_{B} T\right)\right]$ is the probability that the $\mathrm{Mu}$ atom is on the surface and $\zeta=$ $V\left(m k_{B} / 2 \pi \hbar^{2}\right)^{3 / 2} / A S_{0}$ is a constant depending on fundamental constants and material characteristics. This simple model yields excellent agreement for the aerogel data [Fig. 2(a)] (see Table I for fit paramaters).

In contrast, in SBA-15, there is an upturn in $v_{0}^{\mathrm{SBA}}$ below $15 \mathrm{~K}$ [Fig. 2(b)]. This feature is not explained by the simple two-state model; instead, we attribute it to Mu trapped inside the meso- and micropores. Since the $\mathrm{Mu}$ thermal wavelength and the average pore diameter are comparable at those temperatures, quantum effects are expected to play a role, and we tentatively ascribe the low-temperature increase in $\nu_{0}^{\mathrm{SBA}}$ to a compression of the electron wave function inside the pores [30,32]. Excluding this region, the data were fit to Eq. (5) over the temperature range $15-300 \mathrm{~K}$ to allow a direct comparison

TABLE I. Hyperfine frequencies on $\left(v_{s}\right)$ and off $\left(v_{f}\right)$ the surface, binding energy $E_{B}$, and $\zeta$, obtained from a fit to Eq. (5) of the observed $v_{0}(T)$ shown in Fig. 2.

\begin{tabular}{lllcl}
\hline \hline Sample & $v_{f}(\mathrm{MHz})$ & $v_{s}(\mathrm{MHz})$ & $E_{B} / k_{B}(\mathrm{~K})$ & $\zeta\left(\mathrm{K}^{-3 / 2}\right)$ \\
\hline Aerogel & $4461.99(1)$ & $4439.27(7)$ & $42(2)$ & $0.030(1)$ \\
SBA-15 & $4462.67(1)$ & $4439.6(4)$ & $59(3)$ & $0.043(3)$ \\
\hline \hline
\end{tabular}

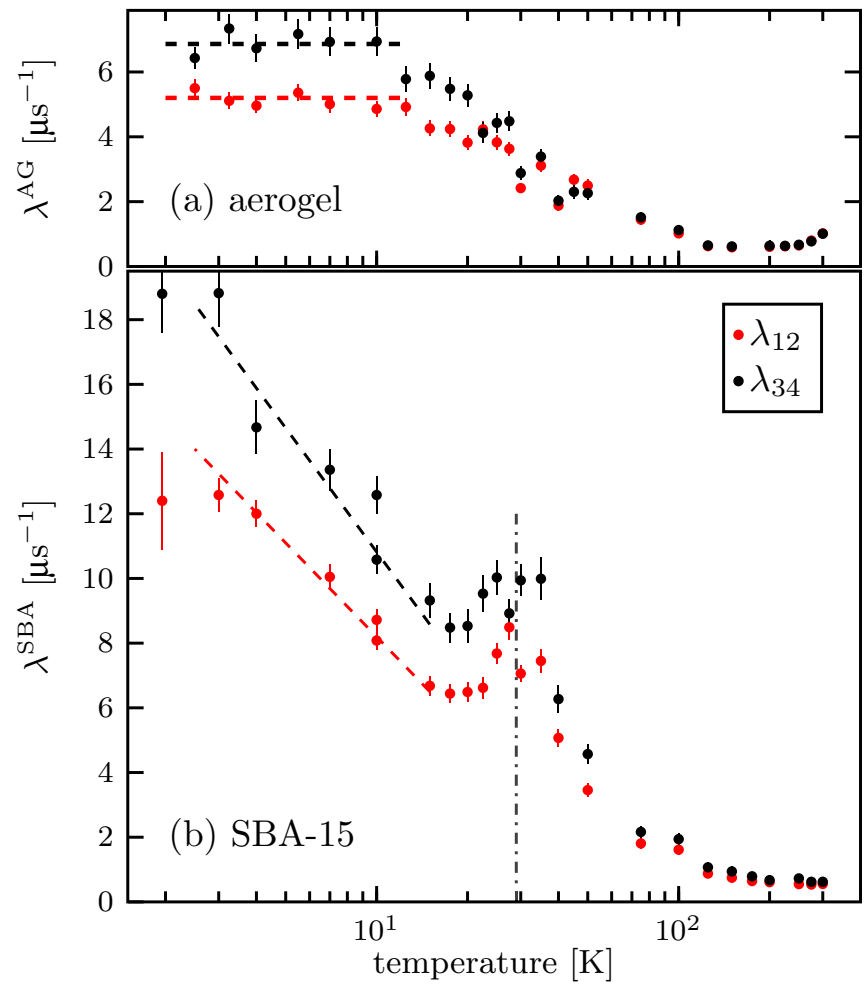

FIG. 3. Relaxation rates $\lambda_{12}$ and $\lambda_{34}$ vs temperature in (a) the aerogel and (b) SBA-15. At low temperatures, $\lambda_{34}$ is larger than $\lambda_{12}$ by a factor of 1.32 (dashed lines), indicating that a distribution of isotropic hyperfine parameters is the dominant source of relaxation.

with the aerogel [orange curve, Fig. 2(b) and Table I]. Additionally, we can qualitatively describe $v_{0}^{\mathrm{SBA}}$ with a three-state model [dashed green line, Fig. 2(b)] by extending Eqs. (4) and (5) to account for an additional surface state with a distinct hyperfine frequency and binding energy. We propose that in SBA-15 such a state may be required to differentiate between $\mathrm{Mu}$ trapped inside micro- rather than mesopores.

Comparable surface binding energies $E_{B}$ are obtained in the aerogel and SBA-15, which is consistent with $\mathrm{Mu}$ binding to silica surfaces in both samples. Above $\sim 100 \mathrm{~K}, \mathrm{Mu}$ spends the vast majority of its lifetime off the surface. In SBA-15, $v_{f}$ is closer to $v_{0}^{\mathrm{vac}}[-0.61(3) \mathrm{MHz}]$ than in the aerogel $[-1.31(1) \mathrm{MHz}]$, indicating that $\mathrm{Mu}$ is making fewer collisions in SBA-15 and thus implying a longer effective mean free path.

The relaxation rates $\lambda$ are shown in Fig. 3. In both samples, $\lambda_{34}$ is significantly larger than $\lambda_{12}$ at low temperatures. Differentiating $v_{12}$ and $v_{34}$ [Eq. (2)] with respect to $v_{0}$ shows that $v_{34}$ is more sensitive to small variations in $v_{0}$, leading to a predictable ratio $\lambda_{34} / \lambda_{12}$ if the line broadening is due to a distribution of isotropic hyperfine parameters on the silica surface:

$$
\lambda_{34} / \lambda_{12}=\left[B / B_{0}+1\right] /\left[B / B_{0}-1\right]=1.32 .
$$

In Fig. 3, the dashed lines are plotted at this ratio, and show good agreement with the data, strongly indicating that a spread in $v_{0}$, or more accurately $v_{s}$, is indeed the main source of broadening in this temperature regime. Parametrizing this 
spread in $v_{s}$ with a Lorentzian of half width $\delta v_{s}$, we estimate $\delta v_{s}(2 \mathrm{~K})=4.6(0.8) \mathrm{MHz}$ for SBA-15 and 2.0(0.3) $\mathrm{MHz}$ in the aerogel [33]. Note that in low transverse fields $(\sim 6 \mathrm{G})$, significantly less damping was observed in SBA-15 [10-12]. This is consistent with our interpretation since a spread in $v_{0}$ causes damping only in large fields, and has no effect on the observable frequencies in low field $\left(v_{12}\right.$ and $v_{23}$, see Ref. [7]); instead, the dominating source of relaxation at low temperatures was identified as hyperfine anisotropy (HFA) $[12,34]$. Since (1) HFA affects both frequencies equally (i.e., $\lambda_{34} / \lambda_{12}=1$ ) and (2) the magnitude of the low-field damping is small compared to the broadening observed here, we conclude that HFA is not the dominant source of relaxation, and thus is not further considered.

The temperature dependence of the relaxation rates in Fig. 3 further support the conclusions drawn from the simple statistical mechanics model described above, although there are indications that the real situation is more complex. In the aerogel [Fig. 3(a)], the decrease of $\lambda^{A G}$ with increasing temperature is consistent with $\mathrm{Mu}$ cycling on and off the surfaces, sampling an increasing number of values from the $v_{s}$ distribution, which leads to motional narrowing. We note that compared to a simple box model, a slower approach to complete motional narrowing is expected for highly porous media with multiple length scales, where a distribution of poorly connected boxes with varying dimensions may be a better description. Thus, for complete motional narrowing, $\mathrm{Mu}$ must rapidly average over all states in all the boxes, requiring higher cycle rates and thus higher temperatures, which leads to a more gradual approach to the motionally narrowed limit. Furthermore, we note that with increasing temperatures, the ratio $\lambda_{34} / \lambda_{12}$ decreases towards unity, indicating that once $\mathrm{Mu}$ becomes mobile, other mechanisms which affect both frequencies equally (e.g., spin exchange with dangling bonds on the silica surface [35]) contribute to the observed relaxation.

Unlike in the aerogel, there is a significant increase in relaxation below $\sim 15 \mathrm{~K}$ in the SBA-15 [Fig. 3(b)]. Again, we attribute this to $\mathrm{Mu}$ confined inside the meso- and micropores, and propose that the broad distribution of hyperfine parameters (as indicated by $\lambda_{34} / \lambda_{12} \approx 1.32$, dashed lines) and the complex line shape [Fig. 1(c), red] are due to a wide distribution of pore diameters. Between 12 and $20 \mathrm{~K}, \lambda^{\text {SBA }}$ levels off at values similar to aerogel $\left(\delta v_{0} \approx 2.5 \mathrm{MHz}\right)$. With rising temperature, $\lambda^{\mathrm{SBA}}$ increases again and has a local maximum near $30 \mathrm{~K}$.

In Eq. (5), we describe the hyperfine constant as a timeweighted average of $v_{f}$ and $v_{s}$. This, however, requires that the cycling rate between the two states is larger than the frequency difference $\Delta v=v_{f}-v_{s}$; cycling rates comparable to $\Delta v$ cause damping, and a further decrease eventually leads to the observation of two distinct signals. Two correlation times $\tau_{s}$ and $\tau_{f}$ describe the cycling process. For $T>100 \mathrm{~K}$, $\alpha \sim 0$ and the free states dominate (i.e., $\tau_{f} \gg \tau_{s}$ ), whereas for $T<10 \mathrm{~K}, \alpha \sim 1$ and only the surface states are relevant. Considerable damping and a local relaxation maximum due to incomplete averaging can only occur when two conditions are met: (1) Both states significantly contribute to the signal, i.e. in the region $\sim 30 \pm 10 \mathrm{~K}(\alpha \sim 0.5 \pm 0.25)$ and (2) the cycle rate is comparable to $\Delta v$ in that region. Since the cycle rate depends on the mean free path and thus on sample morphology, this length-scale requirement for a peak in $\lambda$, while only qualitative, provides a natural explanation for the local maximum in $\lambda^{\mathrm{SBA}}$ near $30 \mathrm{~K}$ [Fig. 3(b)], and its absence in $\lambda^{\mathrm{AG}}$ [Fig. 3(a)], implying a slower cycling rate and thus a longer effective mean free path in the SBA-15 sample. This is consistent with our interpretation above.

Summarizing, we made precise measurements of the $\mathrm{Mu}$ hyperfine interaction in silica aerogel and SBA-15. A simple statistical mechanics model was used to describe $v_{0}(T)$ and estimate the binding energy of $\mathrm{Mu}$ to the silica surface. Above $100 \mathrm{~K}, \mathrm{Mu}$ spends most of its lifetime off the surface in both samples, indicating the possibility to produce thermal $\mathrm{Mu}$ in vacuum well below room temperature. A small reduction in the free hyperfine parameter $v_{f}$ is attributed to collisions with the surface. The shift is smaller in SBA-15, suggesting a larger effective mean free path than in the aerogel, and that SBA-15 or similar materials may have some advantages over aerogel in terms of producing $\mathrm{Mu}$ in vacuum. In particular, ordered porous silica films [36] with a pore spacing small compared to the laser-drilled channels in aerogel [6] $\left(10^{2} \mathrm{~nm}\right.$ vs $10^{5} \mathrm{~nm}$ ) appear to be promising candidates for a high-yield $\mathrm{Mu}$ vacuum source down to $30 \mathrm{~K}$, provided such films can be produced thick enough to stop a surface muon beam and are self-supporting. The different low-temperature behavior of the two samples is attributed to differences in sample morphology, in particular to variations in the mean free path, and the presence of meso- and micropores in SBA-15. A direct measurement of $v_{0}$ in zero applied field [37,38] may resolve the structure of the broad line shape seen in SBA-15, allowing for a detailed study of the properties of confined $\mathrm{Mu}$, and in extension, of confined atomic hydrogen [30,39,40]. The high-transverse-field technique used in this experiment is well suited to characterize the surface interactions and dynamics of $\mathrm{Mu}$ in highly porous materials, and thus may provide a way to test diffusion models for hierarchical and fractal materials where conventional theories for diffusion in homogeneous materials do not apply [15-17].

This work was performed at the Swiss Muon Source $(\mathrm{S} \mu \mathrm{S})$, Paul Scherrer Institute (PSI, Switzerland). M.H.D. acknowledges support from a SBQMI QuEST fellowship. Financial support came from a NSERC Discovery grant to R.F.K. We thank G. Marshall, A. Olin, and G. Beer for discussions and critical reading of the manuscript. The aerogel samples were obtained from the Department of Physics, Chiba University, Chiba 263-8522, Japan [22].
[1] S. G. Karshenboim, Precision physics of simple atoms: QED tests, nuclear structure and fundamental constants, Phys. Rep. 422, 1 (2005).
[2] A. Antognini, P. Crivelli, T. Prokscha, K. S. Khaw, B. Barbiellini, L. Liszkay, K. Kirch, K. Kwuida, E. Morenzoni, F. M. Piegsa, Z. Salman, and A. Suter, Muonium Emission into 
Vacuum from Mesoporous Thin Films at Cryogenic Temperatures, Phys. Rev. Lett. 108, 143401 (2012).

[3] K. S. Khaw, A. Antognini, T. Prokscha, K. Kirch, L. Liszkay, Z. Salman, and P. Crivelli, Spatial confinement of muonium atoms, Phys. Rev. A 94, 022716 (2016).

[4] Y. Miyake, N. Nishida, J. Yoshino, W. Higemoto, E. Torikai, K. Shimomura, Y. Ikedo, N. Kawamura, P. Strasser, S. Makimura, H. Fujimori, K. Nakahara, A. Koda, Y. Kobayashi, K. Nishiyama, R. Kadono, T. Ogitsu, Y. Makida, K. Sasaki, T. Adachi, and K. Nagamine, Ultra slow muon microscopy for nano-science, J. Phys.: Conf. Ser. 302, 012038 (2011).

[5] P. Bakule, Y. Matsuda, Y. Miyake, K. Nagamine, M. Iwasaki, Y. Ikedo, K. Shimomura, P. Strasser, and S. Makimura, Pulsed source of ultra low energy positive muons for near-surface $\mu \mathrm{SR}$ studies, Nucl. Instrum. Methods Phys. Res. Sect. B 266, 335 (2008).

[6] G. A. Beer, Y. Fujiwara, S. Hirota, K. Ishida, M. Iwasaki, S. Kanda, H. Kawai, N. Kawamura, R. Kitamura, S. Lee, W. Lee, G. M. Marshall, T. Mibe, Y. Miyake, S. Okada, K. Olchanski, A. Olin, H. Ohnishi, Y. Oishi, M. Otani et al., Enhancement of muonium emission rate from silica aerogel with a laser-ablated surface, Prog. Theor. Exp. Phys. 2014, $91 \mathrm{C01}$ (2014).

[7] A. Yaouanc and P. Dalmas de Réotier, Muon Spin Rotation, Relaxation, and Resonance (Oxford University Press, Oxford, UK, 2011).

[8] G. Marshall, J. Warren, D. Garner, G. Clark, J. Brewer, and D. Fleming, Production of thermal muonium in the vacuum between the grains of fine silica powders, Phys. Lett. A 65, 351 (1978).

[9] R. F. Kiefl, J. B. Warren, C. J. Oram, G. M. Marshall, J. H. Brewer, D. R. Harshman, and C. W. Clawson, Surface interactions of muonium in oxide powders at low temperatures, Phys. Rev. B 26, 2432 (1982).

[10] M. H. Dehn, D. J. Arseneau, M. D. Bridges, T. Buck, D. L. Cortie, S. P. Cottrell, D. G. Fleming, J. A. Kelly, W. A. MacFarlane, M. J. MacLachlan, G. D. Morris, I. McKenzie, J. Xiao, and R. F. Kiefl, Spin depolarization of muonium in mesoporous silica, J. Phys.: Conf. Ser. 551, 012006 (2014).

[11] M. H. Dehn, D. J. Arseneau, P. Böni, M. D. Bridges, T. Buck, D. L. Cortie, D. G. Fleming, J. A. Kelly, W. A. MacFarlane, M. J. MacLachlan, R. M. L. McFadden, G. D. Morris, P.-X. Wang, J. Xiao, V. M. Zamarion, and R. F. Kiefl, Communication: Chemisorption of muonium on gold nanoparticles: A sensitive new probe of surface magnetism and reactivity, J. Chem. Phys. 145, 181102 (2016).

[12] M. H. Dehn, D. G. Fleming, W. A. MacFarlane, M. J. MacLachlan, V. M. Zamarion, and R. F. Kiefl, Dynamics of anisotropic muonium on silica surfaces explained by Monte Carlo simulation of the muon depolarization, JPS Conf. Proc. 21, 011032 (2018).

[13] P. Bakule, G. A. Beer, D. Contreras, M. Esashi, Y. Fujiwara, Y. Fukao, S. Hirota, H. Iinuma, K. Ishida, M. Iwasaki, T. Kakurai, S. Kanda, H. Kawai, N. Kawamura, G. M. Marshall, H. Masuda, Y. Matsuda, T. Mibe, Y. Miyake, S. Okada et al., Measurement of muonium emission from silica aerogel, Prog. Theor. Exp. Phys. 2013, 103C01 (2013).

[14] D. C. Walker, Muon and Muonium Chemistry (Cambridge University Press, Cambridge, UK, 1983).
[15] S. Seeger, K. H. Hoffmann, and C. Essex, Random walks on random Koch curves, J. Phys. A: Math. Theor. 42, 225002 (2009).

[16] V. Vattipalli, X. Qi, P. J. Dauenhauer, and W. Fan, Long Walks in Hierarchical Porous materials due to combined surface and configurational diffusion, Chem. Mater. 28, 7852 (2016).

[17] M. A. Sadeghi, M. Aghighi, J. Barralet, and J. T. Gostick, Pore network modeling of reaction-diffusion in hierarchical porous particles: The effects of microstructure, Chem. Eng. J. 330, 1002 (2017).

[18] D. A. Kurdyukov, D. A. Eurov, D. A. Kirilenko, J. A Kukushkina, V. V. Sokolov, M. A. Yagovkina, and V. G. Golubev, High-surface area spherical micro-mesoporous silica particles, Microp. Mesop. Mater. 223, 225 (2016).

[19] W. Liu, M. G. Boshier, S. Dhawan, O. van Dyck, P. Egan, X. Fei, M. Grosse Perdekamp, V. W. Hughes, M. Janousch, K. Jungmann, D. Kawall, F. G. Mariam, C. Pillai, R. Prigl, G. zu Putlitz, I. Reinhard, W. Schwarz, P. A. Thompson, and K. A. Woodle, High Precision Measurements of the Ground State Hyperfine Structure Interval of Muonium and of the Muon Magnetic Moment, Phys. Rev. Lett. 82, 711 (1999).

[20] H. Song, R. M. Rioux, J. D. Hoefelmeyer, R. Komor, K. Niesz, M. Grass, P. Yang, and G. A. Somorjai, Hydrothermal growth of mesoporous SBA-15 silica in the presence of PVP-stabilized Pt nanoparticles: Synthesis, characterization, and catalytic properties, J. Am. Chem. Soc. 128, 3027 (2006).

[21] S. Brunauer, P. H. Emmett, and E. Teller, Adsorption of gases in multimolecular layers, J. Am. Chem. Soc. 60, 309 (1938).

[22] M. Tabata, I. Adachi, H. Kawai, T. Sumiyoshi, and H. Yokogawa, Hydrophobic silica aerogel production at KEK, Nucl. Nucl. Instrum. Methods Phys. Res. Sect. A 668, 64 (2012).

[23] R. F. Kiefl, E. Holzschuh, H. Keller, W. Kündig, P. F. Meier, B. D. Patterson, J. W. Schneider, K. W. Blazey, S. L. Rudaz, and A. B. Denison, Decoupling of Muonium in High Transverse Magnetic Fields, Phys. Rev. Lett. 53, 90 (1984).

[24] A. Suter and B. Wojek, MUSRFIT: A free platform-independent framework for $\mu$ SR data Analysis, Phys. Proc. 30, 69 (2012).

[25] A. Adelmann, U. Locans, and A. Suter, The dynamic kernel scheduler-Part 1, Comput. Phys. Commun. 207, 83 (2016).

[26] U. Locans, A. Adelmann, A. Suter, J. Fischer, W. Lustermann, G. Dissertori, and Q. Wang, Real-time computation of parameter fitting and image reconstruction using graphical processing units, Comput. Phys. Commun. 215, 71 (2017).

[27] U. Locans and A. Suter, MUSRFIT - Real time parameter fitting Using GPUs, J. Phys. Soc. Jpn. 21, 011051 (2018).

[28] R. F. Kiefl, B. D. Patterson, E. Holzschuh, W. Odermatt, and D. R. Harshman, Hyperfine splitting of muonium in $\mathrm{SiO}_{2}$ powder, Hyperfine Interact. 18, 563 (1984).

[29] P. Petit, M. Desaintfuscien, and C. Audoin, Temperature dependence of the hydrogen maser wall shift in the temperature range 295-395 K, Metrologia 16, 7 (1980).

[30] E. Roduner, P. W. Percival, P. Han, and D. M. Bartels, Isotope and temperature effects on the hyperfine interaction of atomic hydrogen in liquid water and in ice, J. Chem. Phys. 102, 5989 (1995).

[31] S. B. Crampton, Resonance studies of $\mathrm{H}$ atoms adsorbed on frozen $\mathrm{H}_{2}$ surfaces, J. Phys. Colloq. 41, C7 (1980). 
[32] H. Dilger, E. Roduner, R. Scheuermann, J. Major, M. Schefzik, R. Stößer, M. Päch, and D. Fleming, Mass and temperature effects on the hyperfine coupling of atomic hydrogen isotopes in cages, Physica B 289-290, 482 (2000).

[33] First, Eq. (2) is approximated to $v_{j}=v_{0} / 2 \mp v_{\mu} \mp v_{0}^{2} /\left(8 B \Gamma_{+}\right)$, with the upper (lower) sign for $j=12$ (34). Expansion around a mean hyperfine frequency $\overline{v_{0}}$ yields an expression linear in $v_{0}:\left.v_{j}\left(v_{0}\right)\right|_{v_{0}=\overline{v_{0}}} \approx v_{0}\left(1 \mp B_{0} / B\right) / 2+\overline{v_{0}} /\left(4 B / B_{0}\right) \mp v_{\mu}$. This allows for an estimate for the relaxation rates for a given distribution of $\nu_{0}: \lambda_{j}=\pi\left(1 \mp B_{0} / B\right) \delta v_{0}$.

[34] R. E. Turner and D. R. Harshman, Anisotropic muonium with random hyperfine distortions: A new static relaxation theory, Phys. Rev. B 34, 4467 (1986).

[35] D. R. Harshman, R. Keitel, M. Senba, R. F. Kiefl, E. J. Ansaldo, and J. H. Brewer, Diffusion and trapping of muonium on silica surfaces, Phys. Lett. A 104, 472 (1984).
[36] K. Jiang, Y. Wang, L. Gui, and Y. Tang, Ordered porous films of organically-modified silica prepared by a two-step replicating process, Colloids Surf. A 179, 237 (2001).

[37] E. Holzschuh, W. Kündig, and B. D. Patterson, Direct measurement of the muonium hyperfine frequencies in quartz, Helv. Phys. Acta 54, 552 (1981).

[38] E. Holzschuh, W. Kündig, P. F. Meier, B. D. Patterson, J. P. F. Sellschop, M. C. Stemmet, and H. Appel, Muonium in diamond, Phys. Rev. A 25, 1272 (1982).

[39] S. Patil and Y. Varshni, Properties of confined hydrogen and helium atoms, in Advances in Quantum Chemistry, Vol. 57 (Elsevier, Amsterdam, 2009), pp. 1-24.

[40] N. Aquino, The hydrogen and helium atoms confined in spherical boxes, in Advances in Quantum Chemistry, Vol. 57 (Elsevier, Amsterdam, 2009), pp. 123-171. 